\title{
"Delay-engendered avoidance" reconsidered'
}

\author{
H. Wayne Ludvigson, UNIVERSITY OF TEXAS SOUTHWESTERN MEDICAL SCHOOL \\ James H. MeHose, SOUTHERN ILLINOIS UNIVERSITY
}

\begin{abstract}
Forty-eight female rats received 120 rewarded trials in a 15-ft runway to further investigate the phenomenon of a slow decline in response vigor following the growth of response strength at the outset of training. Contrary to a previous assumption, delay of reward appeared unnecessary for the effect, since a zero-delay group also showed the decline. A delay imposed anterior to the goal did, however, depress response vigor approaching the delay, but the depression was constant over trials. Delay imposed in the goal box had little effect on performance.
\end{abstract}

\section{Problem}

Ludvigson et al (1964) observed that rats, rewarded with food for traversing a long runway, approached a midrunway delay chamber with response speeds which rose steeply within the first 30 trials, and then declined gradually but markedly, only to tend to recover late in training. Coupled with corresponding changes in the incidence of retracing these effects suggested the build-up and attenuation of avoidance behavior. In light of previous research and theorizing regarding a temporal delay of reward, and considering that the avoidance behavior was restricted to the approach to the delay chamber, Ludvigson et al attributed the avoidance to the blocking of the instrumental response by the delay procedure. However, not being originally concerned with such a phenomenon, the study lacked a nonblocked control group, leaving open the possibility that the observed effects might actually be independent of delay. The present study, therefore, was initiated to provide such a control as well as explore the effects of reward magnitude and locus of delay on the phenomenon.

\section{Method}

Forty-eight experimentally-naive female rats of the Charles River CD strain, 58 days of age at the beginning of the study, served as Ss. The Ss were run in an L-shaped double runway, consisting of a 12-in start box (SB), a $31 / 2 \mathrm{ft}$ straight alley $\left(\mathrm{A}_{1}\right)$, a $12-$ in end box $\left(B_{1}\right)$, a second alley $81 / 2 \mathrm{ft}$ in length $\left(A_{2}\right)$, and a second 12-in end box $\left(\mathrm{B}_{2}\right)$ in which all Ss were reinforced. Details of the experimental situation as well as much of the procedure were highly similar to those described previously (Ludvigson et al, 1964).

Starting speeds in $\mathrm{A}_{1}$ and $\mathrm{A}_{2}$ were calculated on the basis of the time lapse between the opening of the $\mathrm{SB}$ or $B_{1}$ start door and $S$ 's crossing an infrared photobeam located 12 in from the start door. The time taken by $S$ in traversing the next 18 in of $A_{1}$ or 78 in of $A_{2}$ to a second photobeam provided the basis for the runningspeed measure.
Twenty-three days prior to the first training day, deprivation schedules were initiated such that daily feeding occurred at the time of day at which S's experimental session ended. Water was available at all times except during a trial in the runway. On Days 18-22 of the preliminary period, Ss were allowed $5 \mathrm{~min}$. of exploration in each alley. Training began on Day 24 . Each $S$ received three trials per day until a total of 120 trials had been administered. The intertrial interval within a day was approximately $25 \mathrm{~min}$.

Four randomly comprised groups of $12 \mathrm{Ss}$ each were assigned the following treatments. Groups SI, $\mathrm{SD}_{1}$, and $\mathrm{SD}_{2}$ all received a "small" reward (one $0.097 \mathrm{gm}$ Noyes pellet) in $\mathrm{B}_{2} ; \mathrm{LD}_{1}$ received a "large" reward (six pellets). Groups $\mathrm{SD}_{1}$ and $\mathrm{LD}_{1}$ were delayed for $10 \mathrm{sec}$. in $\mathrm{B}_{1}$ before being allowed to run to $\mathrm{B}_{2}$; Group $\mathrm{SD}_{2}$ also received a 10-sec. delay, but it was imposed in $\mathrm{B}_{2}$ prior to reward rather than in $B_{1}$. No delay at any point was imposed upon the control group, SI, which received reward immediately upon entrance into $\mathrm{B}_{2}$.

\section{Results}

Mean starting and running speeds for $\mathrm{A}_{1}$ are presented in Fig. 1 and 2, with $A_{2}$ running speeds in Fig. 3. It should be noted that in both running speed measures there is a sharp, statistically-significant "dip" at Block 9. Although bearing a superficial resemblance to an effect observed by Ludvigson et al (1964), it differs from the latter in being highly transitory (limited primarily to a single trial) and present in all groups in both alleys. These considerations, in conjunction with irregular experimental conditions known to have occurred at Block 9, indicated that this dip should be attributed to experimental "error," although it is

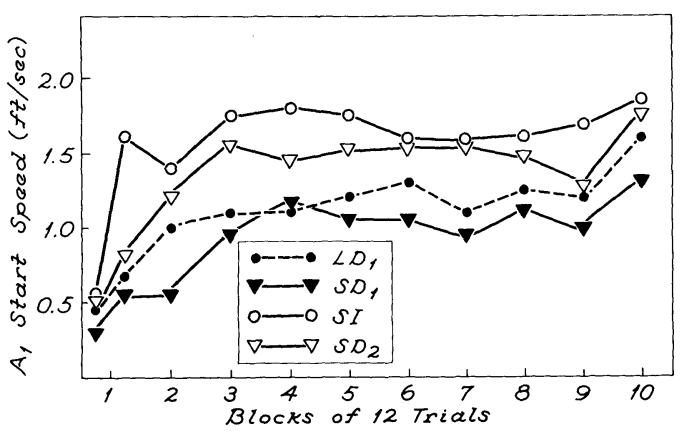

Fig. 1. Mean starting speeds in Alley 1 in 12-trial blocks. (The first block of trials is divided into six-trial points to show initial response strengths.) 


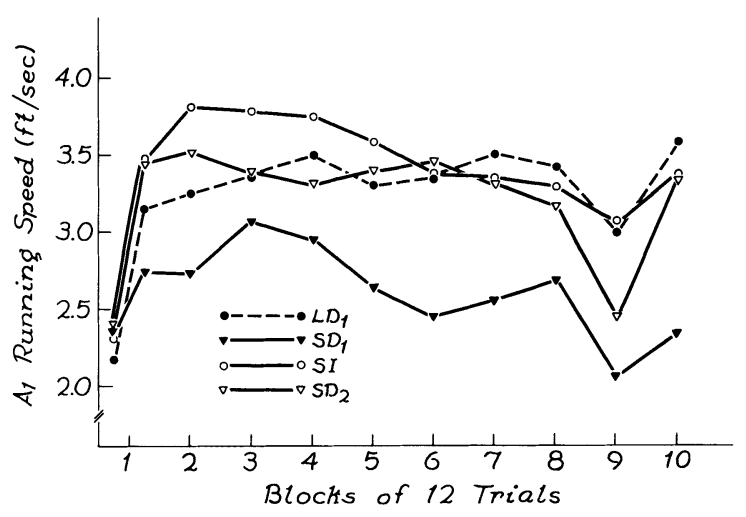

Fig. 2. Mean running speeds in Alley 1.

conceivable that such abrupt drops are genuine (cf. Kendrick, 1958). Of principle interest was the effect of a delay in $\mathrm{B}_{1}$ on $\mathrm{A}_{1}$ performance as revealed by a comparison of Groups $\mathrm{SI}$ and $\mathrm{SD}_{1}$. Analysis over Blocks 3-10 indicated that delay depressed both starting speed, F $(1,22)=11.73 ; \quad p<.005$, and running speed, $F(1,22)=6.91 ; p<.025$, but that there was no suggestion of any change in the extent of the depression over trials, (interaction $\mathrm{F}<1$ for both measures). Running speeds in $\mathrm{A}_{2}$ following the delay were similarly depressed, but not significantly, $F(1,22)=2.62$. For both groups there was a protracted period in which $\mathrm{A}_{1}$ running speeds slowly declined, in much the same manner as in the Ludvigson et al (1964) study. This statement is supported by a significant Blocks effect, $F(7,154)=7.96$; $\mathrm{p}<.001$, as well as the result of a comparison of the mean for Groups $\mathrm{SI}_{\text {and }} \mathrm{SD}_{1}$ at Block 3 with their mean at Block 7, the low point in the Ludvigson et al data, which yielded an $F(1,154)=11.82 ; p<.001$. I may be observed that the other two groups displayed no comparable decremental effect over Blocks 3-7. In addition, it may be observed that the $A_{1}$ start speeds showed very little decremental effect in any group, although the mean for Groups SI and $\mathrm{SD}_{1}$ at Block 7 was significantly lower than their mean at Block $4, F(1,154)=6.85 ; \mathrm{p}<.001$.

In contrast to delay in $\mathrm{B}_{1}$, a delay in the goal box $\left(\mathrm{B}_{2}\right)$ did not cause performance to deviate significantly from a zero-delay condition, since comparisons of Groups SI and $\mathrm{SD}_{2}$ revealed $\mathrm{Fs}<1$ on all three speed measures. This suggests that locus of delay is a relevant variable, a conclusion supported by comparisons of $\mathrm{SD}_{1}$ and $\mathrm{SD}_{2}$ on $\mathrm{A}_{1}$ starting and running speeds, Fs $(1,22)=6.24$ and $3.91 ; \mathrm{p}<.025$ and $<.10$, respectively.

Finally, increased reward magnitude $\left(\mathrm{LD}_{1}\right.$ vs. $\left.\mathrm{SD}_{1}\right)$ had its typical effect of augmenting performance in $\mathrm{A}_{1}$

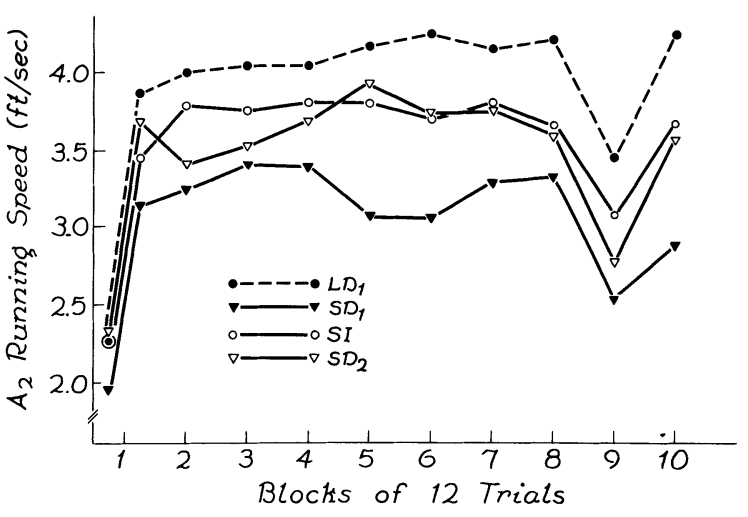

Fig. 3. Mean running speeds in Alley 2.

and $\mathrm{A}_{2}$ running speeds, $\mathrm{F}(1,22)=9.76$ and $7.08 ; \mathrm{p}<.005$ and $<.025$, but not in $A_{1}$ start speeds $(F<1)$.

\section{Diseussion}

The present data replicate the finding of a gradual decremental effect in instrumental conditioning (Ludvigson et al, 1964), but they cast serious doubt upon the assumption that blocking the instumental response by means of a delay of reinforcement is a necessary condition for such an effect. Their relevance for two other effects previously observed, the apparent attenuation of this decremental effect in $A_{1}$ concident with the appearance of a decline in $A_{2}$, is dubious because of the sharp dip late in training.

The gradual loss of response strength observed here may be related to certain other results in instrumental conditioning. The "extinction" reported by Kendrick (1958) bears some resemblance except for the abruptness of its onset and the amount of training prior to its occurrence. An effect in the Marx \& Brownstein (1963) study is even more similar although it cannot be determined whether it was restricted to the anterior portion of the alley as it appeared to be in the present studies.

\section{References}

KENDRICK, D. C. Inhibition with reinforcement (conditioned inhibition). J. exp. Psychol., 1958, 56, 313-318.

LUDVIGSON, H。W., CAUL, W. F。, KORN, J. H., \& McHOSE, J。H Development and attenuation of delay-engendered avoidance behavior. J. exp. Psychol., 1964, 67, 405-411.

MARX, M.H., \& BROWNSTEIN, A.J. Effects of incentive magnitude on running speeds without competing responses in acquisition and extinction. J. exp. Psychol., 1963, 65, 182-189.

Note

1. This investigation was supported in part by Public Health Service Research Grant MH 11029-01 (formerly MH 08852-01) from the National Institute of Mental Health. 